\title{
Efficacité technique et rentabilité économique des méthodes de contrôle culturale et chimique des mauvaises herbes en culture d'oignon (Allium cepa L.) au Nord-Est du Bénin
}

\author{
Saliou Bello ${ }^{1 *}$, Lucien Guillaume Amadji ${ }^{2}$ et Adam Ahanchédé ${ }^{2}$ \\ 1*) Institut National des Recherches Agricoles du Bénin, Centre de Recherches Agricoles d'Agonkanmey, Laboratoire de Défense \\ desCultures, BP 112 Savè,_E-mail : bello_salion@yahoo.fr,Bénin \\ 2) Université d'Abomey-Calavi (UAC), Faculté des Sciences Agronomiques (FSA), Ecole des Sciences et Techniques de \\ Production Végétale (ESTPV), 01 BP 526 Cotonou,E-mail: ahanchedeadam@yahoo.fr/gamadji@yahoo.fr,Bénin \\ Auteur correspondant : Saliou BELLO ${ }^{1 *}, I N R A B / C R A-A / L D C, E-m a i l$ : bello_salion@yahoo.fr, Bénin, Tél : \\ $+22994108119 /+22966614547$
}

Mots-clés : bulbe d'oignon, herbicide, rendement, rentabilité économique, sarclo-binage

Keywords: economic profitability, herbicide, hoe weeding, onion, yield

Publication date 30/11/2019, http://www.m.elewa.org/JAPS

\section{RESUME}

Objectif : Le sarclage pratiqué en culture d'oignon est inefficace contre les Portulacacées et les Cypéracées. La présente étude a permis de comparer les effets des sarclo-binages et d'applications simple et combinée d'herbicides sur la phytotoxicité pour l'oignon, le recouvrement et la biomasse des mauvaises herbes et le rendement de l'oignon au Nord-Est du Bénin.

Méthodologie et résultats : Le dispositif expérimental est un bloc complètement aléatoire installé dans 30 parcelles paysannes représentant chacune une répétition comportant six traitements constitués des pratiques de sarclo-binages exclusifs et combinées à l'application d'herbicides. Les herbicides appliqués sont les sélectifs Agristomp à base de pendiméthaline à la teneur de $400 \mathrm{~g} / 1$ et Dadyochlor à la teneur de $50 \%$ d'acétochlore ainsi que les totaux Round Up et Dady Up à base de glyphosate aux teneurs respectives de 360 $\mathrm{g} / 1$ et de $480 \mathrm{~g} / 1$. Les variables recouvrement, biomasse et rendement ont été soumises respectivement au test $\mathbf{T}$ de Student, à l'analyse de variance à deux facteurs et au test de Tukey.

Une faible phytotoxicité et une meilleure répression de Portulaca quadrifida et de Portulaca oleracea avec Agristomp, une meilleure gestion de Cyperus rotundus et une faible production de biomasse totale avec Round Up ont été observées. Des différences très hautement significatives et un effet synergique ont été observés entre les pratiques de luttes culturale et chimique. Dady Up a engendré le rendement le plus élevé et le plus rentable avec $28,72 \mathrm{t} / \mathrm{ha}$ de bulbes, comparativement aux autres traitements.

Conclusion et application des résultats : La combinaison des sarclo-binages et d'application d'herbicides, plus efficace contre les mauvaises herbes avec un effet synergique et une meilleure rentabilité économique, a été recommandée.

Technical and economical efficacy of hoe-weeding and herbicides application in onion' crop (Allium cepa L.) in the North-East of Benin 


\begin{abstract}
Objective: Weeding in onion is ineffective against Portulacacées and Cyperaceae.. This study compared the effects of weeding, simple and combined herbicide applications on phytotoxicity on onions, weed recovery and biomass, and onion yield in northeastern Benin.Methodology and results: The experimental design is a completely randomized block of 30 replications with six treatments which include exclusive and combined hoe weeding practices to herbicides application. Applied herbicides were selective Agristomp with 400 g/1 of pendimethalin and dadyochlor with $50 \%$ of acetochlor, Round Up and Dady Up with $360 \mathrm{~g} / 1$ and $480 \mathrm{~g} / 1$ of glyphosate respectively. Weed covering and onion yield were submitted to $T$ of Student test, two-way analysis of variance and Tukey test.Low phytotoxicity and better control of Portulaca quadrifida and Portulaca oleracea with Agristomp, better management of Cyperus rotundus and low total biomass production with Round Up were observed.. Very significant differences and a synergistic effect have been observed between the practices of crop and chemical control. Dady Up generated the highest and most cost-effective yield with 28.72 t/ha of bulbs, compared to other treatments.

Finding and applying the results: The combination of weeding and herbicide application, which is more effective against weeds with synergistic effect and improved economic profitability, was recommended.
\end{abstract}

\section{INTRODUCTION}

L'envahissement d'une culture par sa flore associée est la cause de nuisances sur la croissance, le développement et la production. La nuisibilité des mauvaises herbes est liée d'une part à la compétition en eau, en éléments nutritifs, en espace et en lumière (Akobundu, 1987 ; Anonyme, 2002), d'autre part à la libération de composés allélopathiques ou télétoxiques et enfin aux rôles d'hôtes secondaires de différents ravageurs et de vecteurs de maladies joué par la flore nuisible dans les systèmes de cultures. L'envahissement des monocultures de maïs et de sorgho par des poacées annuelles ou vivaces est l'une des causes du plafonnement ou de la diminution des rendements et de l'augmentation des coûts de production. En riziculture irriguée, le coût du désherbage représente $10 \%$ à $20 \%$ des charges de production dans un contexte où le sarclage est souvent délaissé ou tardif (Marty et al., 1977). En Asie, les pertes de rendement en riz pluvial peuvent varier entre $40 \%$ et $100 \%$ si l'enherbement n'est pas correctement géré entre
40 et 60 jours après semis (Anonyme, 2002). Dans les systèmes culturaux africains, ce problème affecte la production de bon nombre de cultures pluviales et maraîchères de première importance. C'est ainsi qu'au Nord-Est du Bénin, les mauvaises herbes constituent une contrainte majeure dans les systèmes de cultures maraîchères en général et de l'oignon en particulier (Gotoéchan-Hodonou et Savi, 2001 ; Gnanglè et al., 2002 ; INRAB, 2005 ; Bello, 2012 ; Bello, 2013 ; Bello et al., 2013a ; Bello et al., 2013b). Dans cette région, l'enherbement affecte la production de semences d'oignon dans 5\% des exploitations (Gotoéchan-Hodonou et Savi, 2001) et la production de bulbes d'oignon dans 90\% des exploitations (Bello, 2012). Par contre au SudBénin où la situation de l'enherbement des parcelles d'oignon n'est pas aussi inquiétante, Assogba-Komlan et al. (2006) ont recommandé le sarclage des planches sableuses à partir de la deuxième semaine après le repiquage. Les sarclages manuels associés à l'arrachage, au 
ramassage et à l'enfouissement de la biomasse sarclée sont inefficaces contre Portulaca quadrifida, Portulaca oleracea et Cyperus rotundus dans les casiers d'oignon irrigués au Nord-Est du Bénin (Bello, 2012). Tous les producteurs s'accordaient à affirmer que ces trois espèces sont assez compétitives pour la culture d'oignon, malgré que des désherbages soient réalisés à 15,45 et 60 jours après repiquage (Bello et al., 2004). En effet, le désherbage manuel nettoie les parcelles infestées de Cyperus rotundus, tout en favorisant la multiplication de l'espèce (Dembélé et al., 1996). Cette technique permet aussi, d'après N'diaye et al. (1997), de fragmenter et/ou de disperser les organes de dissémination et de favoriser les conditions de multiplication de Cyperus rotundus, de Portulaca oleracea et de Portulaca quadrifida. La préparation du sol et le sarclage manuel, la rotation avec les cultures céréalières, maïs, riz, sorgho et mil (Anonyme, 2002), ainsi que le faux-semis ou la pré-irrigation (Marnotte, 2000) sont les seules méthodes très couramment utilisées par les producteurs de la zone d'étude. Néanmoins, l'application d'herbicides peut être envisagée (Valdez, 1994) pour élargir la gamme de méthodes de lutte et permettre leur utilisation combinée. Dembélé et al. (1996), puis N'diaye et al. (1997) ont eu le mérite de recommander trois applications de $4 \mathrm{l} /$ ha de glyphosate à des

\section{MATERIEL ET METHODES}

3.1 Zone d'étude: La zone d'étude est localisée dans le département de l'Alibori située au Nord-Est du Bénin, entre les parallèles $11^{\circ} 30$ et $12^{\circ} 30$ de latitude Nord et les méridiens $2^{\circ} 43$ et $3^{\circ} 20$ de longitude Est. Elle est représentée par les villages de Toumboutou et de Kargui situés respectivement dans les communes de Malanville et de Karimama au Nord-Est du Bénin (figures $1 \mathrm{a}$ et 1b). Le climat était de type soudano-sahélien avec une pluviométrie annuelle variable de 800 à 900 $\mathrm{mm}$. Les sols avaient une texture sablo-argileuse à sablo-limoneuse. pousses en pleine végétation et au système de rhizomes et de tubercules intacts, à environ 40 jours après la récolte (JAR) de la culture, pour réduire la population de Cyperus rotundus à un seuil de nuisibilité où elle ne cause plus de dégâts à la plante cultivée. Les formulations d'herbicides à base de glyphosate aux teneurs de $360 \mathrm{~g} / 1$ et de $700 \mathrm{~g} / 1$ avaient été homologuées et recommandés sur le maïs et le coton (CNAC, 2012). De même, les herbicides sélectifs Dadyochlor à base d'acétochlore à la teneur de 50\% et d'Agristomp à base de $400 \mathrm{~g} / 1$ de pendiméthaline ont été recommandés sur des mauvaises herbes difficiles à maitriser telles que Cyperus rotundus dans les cultures céréalières maïs, riz et maraîchères notamment la tomate et le piment (Anonyme, 1995 ; Aboué, 2005 ; Marnotte et Téhia, 1991 ; Anonyme, 2006a ; Anonyme, 2006b ; Anonyme, 2008a ; Anonyme, 2008b). Toutefois, des efforts méritent d'être faits pour élaborer des recommandations d'emploi de ces herbicides en culture d'oignon. La présenté vise à évaluer l'effet sur la gestion de l'enherbement, le rendement en bulbes d'oignon et la rentabilité économique des pratiques simples et combinées de sarclo-binages répétés et d'application de deux herbicides totaux à base de glyphosate et de deux herbicides sélectifs à base d'acétochlore et de pendiméthaline.

\subsection{Matériel végétal et conduite de la} culture: Le matériel végétal utilisé était la variété locale d'oignon de couleur rouge violacé, appelée localement "Aloubassa ». L'oignon était cultivé en casiers de $3 \mathrm{~m}^{2}$ irrigués selon un mode d'irrigation par gravité.

3.3 Dispositif expérimental : Le dispositif expérimental était un bloc complètement aléatoire (BCA) installé auprès de 30 producteurs comme répétitions et comportant six parcelles élémentaires, représentant chacun un traitement appliqué dans 10 casiers, l'équivalent de $30 \mathrm{~m}^{2}$ de superficie. Les six 
traitements considérés comprenaient deux pratiques paysannes de sarclo-binages manuels répétés au cours du cycle végétatif de l'oignon, l'application de deux herbicides totaux et l'application de deux herbicides sélectifs combinés aux sarclo-binages. La pratique paysanne des sarclo-binages, qui est celle dominante dans la zone d'étude et qui a consisté en trois opérations effectuées à 15, 45 et $60 \mathrm{JAR}$, est réalisée sur sols faiblement envahis et sur sols fortement envahis. Elle est associée au besoin à des arrachages manuels suivis d'un ramassage et d'un enfouissement de la biomasse sarclée hors de la parcelle de culture. Les traitements herbicides combinés aux sarclobinages étaient réalisés sur les parcelles fortement enherbées, aux doses recommandées par les firmes fabricantes (tableau 1).

Tableau 1 : Description et modes de réalisation des méthodes de lutte testées

\begin{tabular}{|c|c|c|}
\hline Pratiques de contrôle & Description & Mode de réalisation \\
\hline \multicolumn{3}{|c|}{ Sarclo-binages manuels répétés, réalisés à 15, 45 et 60 JAR sur : } \\
\hline $\begin{array}{l}\text { Parcelle faiblement envahie } \\
\text { (densité de mauvaises herbes } \leq 4 \\
\text { plants } / \mathrm{m}^{2}(\mathrm{PFaE})\end{array}$ & \multirow{2}{*}{$\begin{array}{l}\text { Pratique paysanne de sarclage et de } \\
\text { binage réalisés respectivement à la houe } \\
\text { et à la binette }\end{array}$} & \multirow{2}{*}{$\begin{array}{l}\text { Trois sarclo-binages manuels effectués à } \\
15,45 \text { et } 60 \text { jours après repiquage (JAR) } \\
\text { sur }\end{array}$} \\
\hline $\begin{array}{l}\text { Parcelle fortement envahie (densité } \\
\text { de mauvaises herbes }>\text { à } 5 \text { et } \geq 8 \\
\text { plants } / \mathrm{m}^{2}(\mathrm{PFOE})\end{array}$ & & \\
\hline \multicolumn{3}{|c|}{$\begin{array}{l}\text { Sarclo-binages manuels réalisés à 15, } 45 \text { et } 60 \text { JAR sur parcelle fortement envahie (densité de mauvaises herbes }> \\
\qquad \dot{a} 5 \text { et } \geq 8 \text { plants } / \mathrm{m}^{2} \text { ) combinés à l'application d'herbicide : }\end{array}$} \\
\hline Round Up (Round Up) & $\begin{array}{l}360 \mathrm{~g} / 1 \text { de glyphosate à la dose de } 6 \\
\text { l/ha. }\end{array}$ & $\begin{array}{l}\text { - Application de l'herbicide à } 21 \text { jours } \\
\text { avant la confection des casiers } \\
\text { - Pulvérisation de bouillie contenant } 18 \\
\text { ml du produit dilués dans } 1,21 \text { d'eau } \\
\text { pour traiter } 30 \mathrm{~m}^{2}\end{array}$ \\
\hline Dady Up (Dady Up) & $480 \mathrm{~g} / 1$ de glyphosate à la dose de 8 1/ha & $\begin{array}{l}\text { - Application de l'herbicide à } 21 \text { jours } \\
\text { avant la confection des casiers } \\
\text { - Pulvérisation de bouillie contenant } 24 \\
\text { ml du produit dilués dans } 1,21 \text { d'eau } \\
\text { pour traiter } 30 \mathrm{~m}^{2}\end{array}$ \\
\hline Agristomp $500 \mathrm{E}$ (Stomp) & $\begin{array}{l}400 \mathrm{~g} / \mathrm{l} \text { de pendiméthaline sous forme } \\
\text { de concentré émulsionnable à la dose de } \\
2,5 \mathrm{l} / \mathrm{ha}\end{array}$ & $\begin{array}{l}\text { - Application de l'herbicide après la } \\
\text { confection des casiers, et à la pré- } \\
\text { irrigation des casiers } \\
\text { - Repiquage des plantules d'oignon le } \\
\text { même jour } \\
\text { - Pulvérisation de bouillie contenant } 7,5 \\
\text { ml du produit dilués dans } 1,21 \text { d'eau } \\
\text { pour traiter } 30 \mathrm{~m}^{2}\end{array}$ \\
\hline Dadyochlor (Dadyochlor) & $\begin{array}{l}50 \% \text { d'acétochlore sous forme de } \\
\text { concentré émulsionnable, à la dose de } \\
1,5 \text { l/ha. }\end{array}$ & $\begin{array}{l}\text { - Application de l'herbicide après la } \\
\text { confection des casiers, et à la pré- } \\
\text { irrigation des casiers } \\
\text { - Repiquage des plantules d'oignon le } \\
\text { même jour } \\
\text { - Pulvérisation de bouillie contenant } 4,5 \\
\text { ml du dilués dans } 1,21 \text { d'eau pour traiter } \\
30 \mathrm{~m}^{2}\end{array}$ \\
\hline
\end{tabular}

Les parcelles faiblement envahies (PFaE) avaient une densité de mauvaises herbes au plus égale à quatre (04) plants $/ \mathrm{m}^{2}$ tandis que celle fortement envahies (PFoE) avait une densité 
variable de cinq (05) à huit (08) plants $/ \mathrm{m}^{2}$. Les quatre (04) herbicides testés étaient constitués de (i) l'herbicide total Round Up à base de glyphosate à la teneur de $360 \mathrm{~g} / \mathrm{l}$, (ii) l'herbicide sélectif Agristomp à base de pendiméthaline à la teneur de $400 \mathrm{~g} / \mathrm{l}$, (iii) l'herbicide total Dady Up à base de glyphosate à la teneur de $480 \mathrm{~g} / \mathrm{l}$, et (iv) l'herbicide sélectif Dadyochlor à base d'acétochlore à la teneur de 50\%. Aucun traitement exclusif à base d'herbicide et aucun traitement non sarclé n'étaient réalisé en raison du niveau d'enherbement élevé qui est observé sur certaines parcelles en rapport avec la diversité, l'abondance et la maîtrise difficile des espèces de mauvaises herbes présentes (Bello, 2013 ; Bello et al., 2013a ; Bello et al., 2013b).

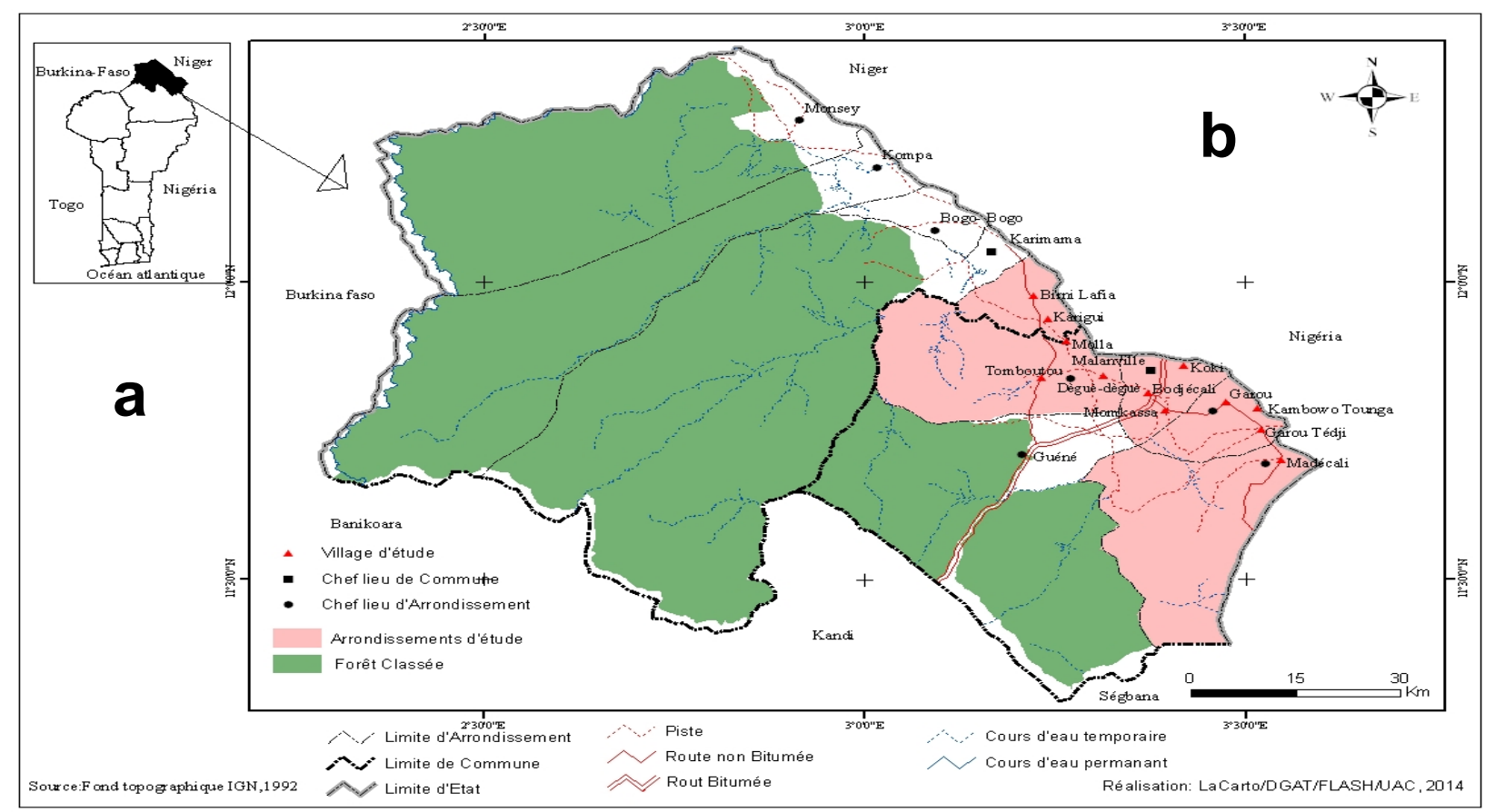

Figure 1 : Carte du Bénin montrant la zone d'étude représentée par les communes de Malanville et de Karimama (a) et la localisation géographique des villages d'étude (b)

Source : Bello (2013)

3.4 Collecte des données : Les paramètres observés et analysés étaient la phytotoxicité de l'herbicide et l'effet destructeur des plantules lié au sarclage, le recouvrement et la biomasse des mauvaises herbes et le rendement en bulbes d'oignon. La phytotoxicité et l'effet destructeur des traitements, évalués à 5 et à 15 jours après repiquage (JAR), étaient calculés par la formule : $100 *$ (nombre total de plants morts ou détruits)/nombre total de plants qui ont repris après le repiquage.

Le recouvrement des mauvaises herbes, qui représente la proportion (\%) de la superficie totale du sol occupée par celles-ci, est mesuré à $5,15,30,45,60,75$ et 90 JAR. L'évaluation était faite séparément pour Cyperus rotundus, et pour Portulaca oleracea et Portulaca quadrifida ensemble. Ces trois espèces sont considérées comme les plus importantes mauvaises herbes rencontrées en culture d'oignon, de par leur fréquence, abondance et dominance (Bello, 2013 ; Bello et al., 2013a ; Bello et al., 2013b). L'appréciation du recouvrement est faite avant les sarclages aux sept périodes ci-dessus décrites au moyen de l'échelle de la Commission des Essais Biologiques (CEB) modifiée par 
Marnotte et al. (2004). Les notes, variables de 1 à 9 correspondaient à des pourcentages de recouvrement du sol comme suit : $1: 1 \%$ à $4 \%$; $2: 4,1 \%$ à $11 \% ; 3: 11,1 \%$ à $22,5 \% ; 4: 22,6 \%$ à $40 \% ; 5: 40,1 \%$ à $60 \% ; 6: 60,1 \%$ à $77,5 \% ; 7$ : $77,51 \%$ à $89 \% ; 8: 89,1 \%$ à $96,5 \% ; 9: 96,51 \%$ à $100 \%$. Les niveaux d'enherbement des mauvaises herbes sont caractérisés par les médianes des notes qui représentaient, mieux que les moyennes, la valeur centrale d'une distribution (Snedecor et Cochran, 1971). Des relevés floristiques parcellaires sont réalisés pour identifier la présence de Portulaca quadrifida et de Portulaca oleracea ainsi que de Cyperus rotundus auxquelles sont attribuées les notes de recouvrement. La biomasse sarclée est pesée à l'aide d'un peson à pile de portée maximale 3 $\mathrm{kg}$, suivant un tri séparé pour Cyperus rotundus seul, Portulaca oleracea et Portulaca quadrifida ensemble et pour les autres espèces. L'évaluation est faite après les sarclages effectués à 15, 45 et 60 JAR. Les échantillons sont séchés jusqu'à l'obtention de poids sec constant au laboratoire. Les biomasses spécifiques de ces trois groupes d'espèces ainsi que leurs valeurs totales sont calculées et exprimées en $\mathrm{kg} / \mathrm{ha}$ de matière sèche. Les mesures de poids parcellaires de bulbes d'oignon sont réalisées à la maturité de l'oignon, qui a lieu entre 90 et $100 \mathrm{JAR}$, à l'aide d'une balance mécanique de $25 \mathrm{~kg}$ de portée. Les données de poids mesurés sont rapportées

\section{RESULTATS}

4.1 Effets phytotoxique et/ou destructeur des pratiques sur les plantules d'oignon: Les sarclo-binages ont souvent engendré la destruction des plantules par l'inobservance de mesures de prudence de la part des producteurs ou de leurs manœuvres lors de l'opération. La toxicité des herbicides sur les plantules d'oignon était imputable aux effets physiologiques des herbicides appliqués. Sur les parcelles qui ont reçu l'application d'herbicide, la combinaison de ces pratiques en $t /$ ha de bulbes frais. Ces différents paramètres sont mesurés sur chaque parcelle élémentaire, dans cinq carrés d'observation de 1 $\mathrm{m}^{2}$, disposés à raison de deux sur chaque diagonale et un au centre de la parcelle. Les appréciations paysannes et le devenir des résultats sont évalués grâce à des entretiens semi-structurés lors d'une assemblée villageoise de restitution et d'évaluation des résultats qui a réuni les participants et les non participants à l'essai.

3.5 Analyse statistique des données : Les données de phytotoxicité des herbicides et d'effet destructeur des sarclo-binages et la variable recouvrement sont soumises à l'analyse de variance uni-variée sur mesures répétées à deux facteurs de classification que sont la méthode de lutte et la période d'observation. Les données de biomasse totale obtenue au cours des trois dates de sarclage sont soumises à une analyse de variance à un seul facteur qu'est la méthode de lutte. Une analyse de variance uni-variée à un facteur en blocs complètement randomisés était effectuée pour la variable rendement. Pour chaque variable, ces analyses de variance ont servi à déterminer les probabilités de signification des facteurs au seuil de 5\%. La comparaison des moyennes des variables phytotoxicité, recouvrement, biomasse et rendement est effectuée avec le test de Tukey au seuil de 5\%. Le logiciel Statistik 8 était utilisé pour ces différents tests statistiques.

culturales et chimiques pouvait engendrer à la fois les deux types d'effets. Le tableau 2 présente les nombres et les pourcentages (entre parenthèses) de plants/casier de $3 \mathrm{~m}^{2}$ qui ont péris ou ont été détruits du fait des différentes méthodes de lutte. L'herbicide Round Up a créé l'effet toxique le plus important, avec 7\% des plantules d'oignon, suivi de Agristomp pour 3\% des plantules. Les sarclo-binages sur parcelles fortement envahies et faiblement envahies ont suivi avec $3 \%$ et $1 \%$ des plants respectivement. 
Tableau 2 : Synthèse des résultats d'analyse statistique des effets de phytotoxicité et létal (nombre et \% de plants par casier de $3 \mathrm{~m}^{2}$ ) liés aux différentes méthodes de lutte

\begin{tabular}{|c|c|c|c|}
\hline \multirow{2}{*}{ Méthodes de contrôle testées } & \multicolumn{3}{|c|}{$\begin{array}{l}\text { Phytotoxicité } \\
\text { (nombre et \% de plants repris à } 5 \text { JAR et cumulés à 15 JAR) }\end{array}$} \\
\hline & à $5 \mathrm{JAR}$ & \multicolumn{2}{|c|}{ cumulée à 15 JAR } \\
\hline \multicolumn{4}{|l|}{ Sarclo-binages répétés sur } \\
\hline Parcelle faiblement envahie (PFaE) & $0,6 \mathrm{~dB}(0,9)$ & \multicolumn{2}{|c|}{$0,7 \mathrm{dA}(1,1)$} \\
\hline Fortement envahie (PFoE) & $2,9 \mathrm{bA}(3,5)$ & \multicolumn{2}{|c|}{$3,1 \mathrm{bA}(3,8)$} \\
\hline \multicolumn{4}{|c|}{$\begin{array}{l}\text { Application d'herbicide sur parcelle fortement envahie (densité de mauvaises herbes }>\text { à } 4 \text { plants } / \mathrm{m}^{2} \text { ) combinée aux } \\
\text { sarclobinages répétés }\end{array}$} \\
\hline Agristomp (Stomp) & \multicolumn{2}{|c|}{$2,3 \mathrm{cA}(2,9)$} & $2,2 \mathrm{cA}(2,7)$ \\
\hline Round Up (Round Up) & \multicolumn{2}{|l|}{$5,3 \mathrm{aA}(6,5)$} & $5,5 \mathrm{aA}(6,7)$ \\
\hline Moyenne & \multicolumn{2}{|l|}{$2,78(3,5)$} & $2,88(3,6)$ \\
\hline Ecart type & \multicolumn{2}{|l|}{$1,96(2,3)$} & $2,01(2,4)$ \\
\hline Source de variation & Méthode de contrôle & Période & Méthode * période \\
\hline Ddl & 26 & 1 & 26 \\
\hline Probabilité $(p<F)$ & $0,00^{* *}$ & $0,01 *$ & $0,02 *$ \\
\hline
\end{tabular}

Les valeurs moyennes suivies des différentes lettres a, b, c et d sont significativement différentes au seuil de signification de 5\% d'après le test de Tukey pour la colonne considérée.

Les lettres A et B indiquent une différence significative entre les moyennes des doses aux deux périodes végétatives de l'oignon.

**: très significatif; $*$ significatif

\subsection{Evolution du recouvrement des mauvaises herbes}

4.2.1 Recouvrement de Portulaca oleracea et de Portulaca quadrifida: La figure 2 présente l'évolution du recouvrement de Portulaca oleracea et de Portulaca quadrifida considérés ensemble dans les casiers d'oignon en fonction des méthodes de lutte. Sur les parcelles faiblement et fortement envahies, le recouvrement des deux Portulacacées a varié entre
$72 \%$ et $75 \%$ au cours du cycle végétatif de l'oignon, avec un coefficient de variation (CV) compris entre $15 \%$ et $28 \%$. Sur les parcelles traitées au Round Up, ces deux espèces de mauvaises herbes étaient moins agressives que sur les parcelles faiblement et fortement envahies, avec un taux de recouvrement qui a varié pour l'herbicide Agristomp, de $61 \%$ à 5 JAR à $51 \%$ à 90 JAR. 


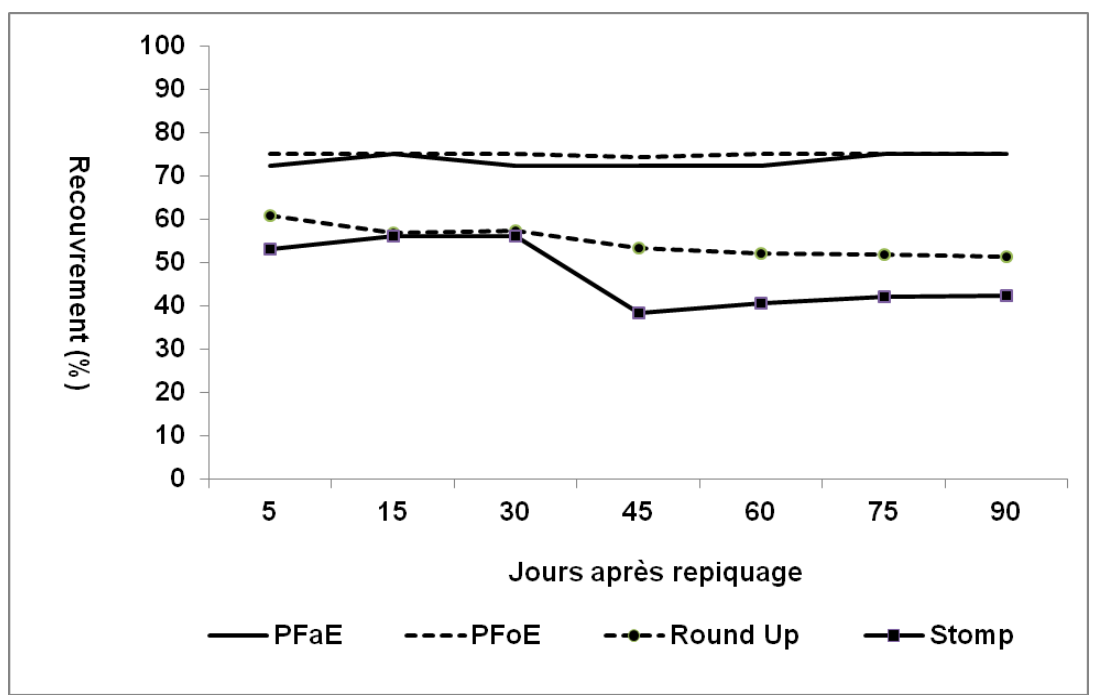

Figure 2 : Evolution du recouvrement (\%) de Portulaca quadrifida et de Portulaca oleracea au cours du cycle végétatif de l'oignon (JAR)

\subsubsection{Recouvrement de Cyperus compris entre $62 \%$ et $72 \%$, contre $69 \%$ à $75 \%$} rotundus : La figure 3 présente l'évolution du sur les parcelles faiblement envahies, avec un recouvrement de Cyperus rotundus. Sur les coefficient de variation (CV) qui s'est accru de parcelles fortement envahies, cette espèce de 16,78\% à 30,23\%.

mauvaise herbe a présenté un recouvrement

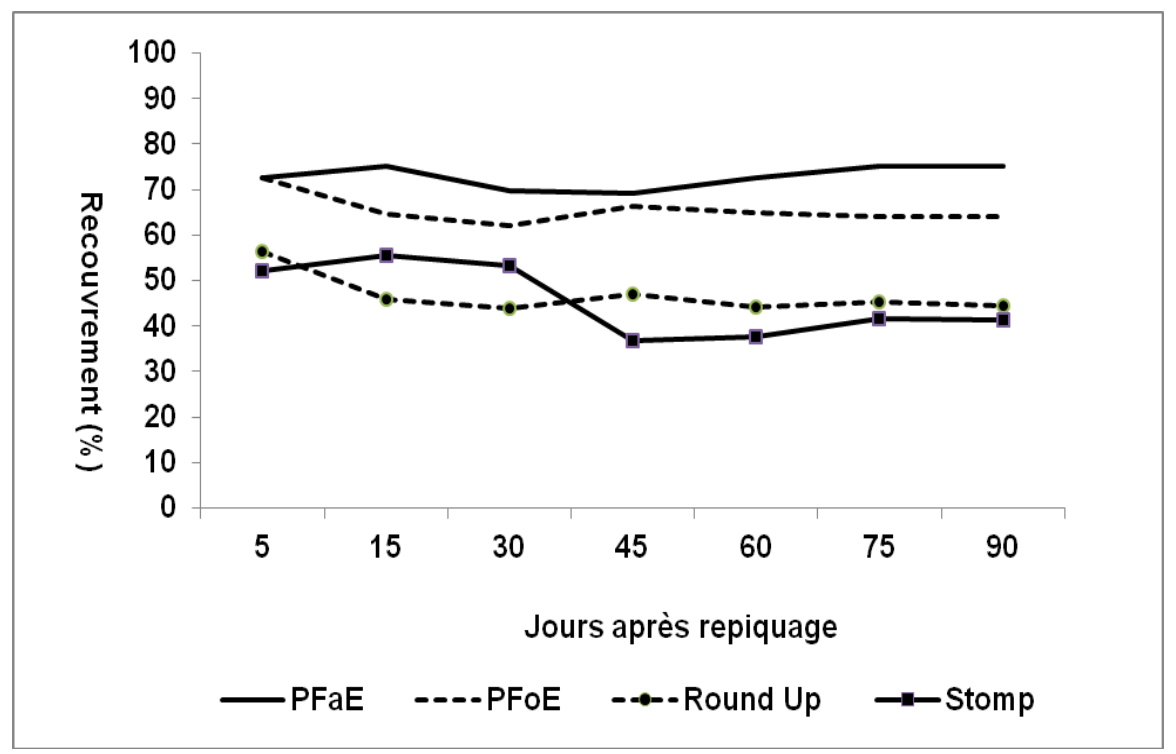

Figure 3 : Evolution du recouvrement (\%) de Cyperus rotundus au cours du cycle végétatif de l'oignon (JAR)

Le Round Up a montré une plus forte efficacité à partir de $45 \mathrm{JAR}$ avec une réduction du recouvrement de $56 \%$ à $44 \%$ contre $52 \%$ à
41\% pour Agristomp, comparativement aux parcelles fortement envahies où le recouvrement s'est accru de $72 \%$ à $75 \%$. Les 
résultats d'analyse de variance des données de recouvrement présentés au tableau 3 indiquaient une forte signification des facteurs traitement $(p<0,001)$, espèce $(p<0,001)$ et période végétative $(\mathrm{p}<0,001)$ en nombre de jours après repiquage (JAR). Une interaction très hautement significative des facteurs traitement et JAR d'une part, et des facteurs traitement et espèce d'autre part est observée ( $\mathrm{p}$ $<0,001)$. Le recouvrement de Portulaca oleracea et de Portulaca quadrifida, de $62,57 \%$, est plus élevé que celui de Cyperus rotundus ( $\mathrm{p}<0,001)$, estimé à $57,62 \%$. Aucune différence statistique significative n'est observée de 5 JAR à $90 \mathrm{JAR}$, entre les recouvrements moyens calculés pour les deux espèces sur les parcelles faiblement envahies et sur les parcelles fortement envahies, toutes deux non traitées à l'herbicide. Cette constatation était similaire pour les parcelles fortement envahies traitées au Round Up et celles traitées avec Agristomp. Toutefois, pour l'ensemble des traitements, les valeurs de recouvrement ont présenté des différences très hautement significatives d'une période à une autre. Le plus fort recouvrement est obtenu à $15 \mathrm{JAR}$ et le plus faible à $45 \mathrm{JAR}$ et à $60 \mathrm{JAR}$.

4.3 Effet des méthodes de lutte sur la production de biomasse de mauvaises herbes: Aucune différence significative n'est observée entre les moyennes de biomasse des mauvaises herbes pour les deux campagnes ( $p$ $=0,065)$. Quelle que soit la méthode de lutte considérée, la biomasse de Cyperus rotundus, bien que son recouvrement soit plus faible, est plus importante que celle de Portulaca oleracea et de Portulaca quadrifida (tableau 4). Des différences significatives sont observées entre les biomasses obtenues avec les différentes méthodes de lutte $(0,000 \leq \mathrm{p} \leq 0,006)$. Par ordre d'importance décroissante de la biomasse produite, la pratique paysanne des sarclo-binages sur parcelles fortement envahies (PFoE) venait en tête, suivie des sarclo-binages combinés à Agristomp 500 E, puis de Round Up et enfin des sarclo-binages sur parcelles faiblement envahies (PFaE). Pour la biomasse totale, les parcelles fortement envahies non traitées venaient en tête, suivies des parcelles traitées au Round up et enfin des parcelles traitées à Agristomp et des parcelles faiblement envahies non traitées.

4.4 Effet des méthodes de lutte sur la production de bulbes d'oignon : La réponse du rendement aux différentes méthodes de lutte testées contre les mauvaises herbes est présentée à la figure 4. Les rendements moyens n'ont montré aucune différence significative $(p$ $>0,05)$ entre les valeurs obtenues pour Round Up et Agristomp au cours des deux campagnes. Par contre, le test était très hautement significatif $(\mathrm{p}<0,001)$ et des différences significatives sont observées entre les moyennes. 
Tableau 3 : Synthèse des résultats de l'analyse statistique du recouvrement de Portulaca oleracea et de Portulaca quadrfida ensemble (P) et de Cyperus rotundus (C) à différentes périodes végétatives de l'oignon (JAR)

\begin{tabular}{|c|c|c|c|c|c|c|c|c|c|c|c|c|c|c|c|}
\hline \multirow{3}{*}{$\begin{array}{l}\text { Traitements } \\
\qquad(\mathrm{T})\end{array}$} & \multicolumn{14}{|c|}{ Jours après repiquage (JAR) } & \multirow{3}{*}{ Moyenne } \\
\hline & \multicolumn{2}{|c|}{5} & \multicolumn{2}{|c|}{15} & \multicolumn{2}{|c|}{30} & \multicolumn{2}{|c|}{45} & \multicolumn{2}{|c|}{60} & \multicolumn{2}{|c|}{75} & \multicolumn{2}{|c|}{90} & \\
\hline & $\mathbf{C}$ & $\mathbf{P}$ & $\mathbf{C}$ & $\mathbf{P}$ & $\mathbf{C}$ & $\mathbf{P}$ & $\mathrm{C}$ & $\mathbf{P}$ & $\mathbf{C}$ & $\mathbf{P}$ & $\mathbf{C}$ & $\mathbf{P}$ & $\mathbf{C}$ & $\mathbf{P}$ & \\
\hline PFaE & 72,45 & 72,45 & 75,00 & 75,00 & 69,83 & 72,45 & 69,08 & 72,45 & 72,45 & 72,45 & 75,00 & 75,00 & 75,00 & 75,00 & $\begin{array}{c}73,12 \pm \\
0,46 a\end{array}$ \\
\hline PFoE & 72,45 & 75,00 & 64,65 & 75,00 & 62,10 & 75,00 & 66,45 & 74,25 & 64,95 & 75,00 & 64,13 & 75,00 & 64,13 & 75,00 & $\begin{array}{c}70,22 \pm \\
0,46 \mathrm{a}\end{array}$ \\
\hline Round Up & 56,33 & 60,83 & 45,83 & 56,78 & 43,80 & 57,38 & 47,10 & 53,40 & 44,10 & 52,05 & 45,23 & 51,83 & 44,40 & 51,45 & $\begin{array}{c}50,75 \pm \\
0,46 \mathrm{~b}\end{array}$ \\
\hline Stomp & 52,20 & 53,18 & 55,58 & 56,10 & 53,33 & 56,03 & 36,83 & 38,48 & 37,65 & 40,73 & 41,70 & 42,23 & 41,48 & 42,45 & $\begin{array}{c}46,25 \pm \\
0,46 b\end{array}$ \\
\hline $\begin{array}{l}\text { Moyenne } \\
\text { espèces }\end{array}$ & $63,36 \mathrm{~B}$ & $65,36 \mathrm{~A}$ & $60,26 \mathrm{~B}$ & $65,72 \mathrm{~A}$ & $57,26 \mathrm{~B}$ & $65,21 \mathrm{~A}$ & $54,86 \mathrm{~B}$ & $59,64 \mathrm{~A}$ & $54,79 B$ & $60,06 \mathrm{~A}$ & $56,51 \mathrm{~B}$ & $61,02 \mathrm{~A}$ & $56,25 \mathrm{~B}$ & $60,97 \mathrm{~A}$ & $\begin{array}{c}60,09 \pm \\
13,55\end{array}$ \\
\hline $\begin{array}{ll}\mathrm{CV} & (\%) \\
\text { espèces } & \end{array}$ & 16,78 & 15,60 & 20,70 & 16,31 & 19,60 & 15,18 & 28,27 & 28,46 & 30,23 & 27,44 & 27,91 & 27,23 & 28,52 & 27,23 & 19,54 \\
\hline $\begin{array}{l}\text { Moyenne } \\
\text { JAR }\end{array}$ & $64,36 \pm$ & $0,61 \mathrm{~A}$ & $62,99 \pm$ & $0,61 \mathrm{AB}$ & $61,24 \pm$ & $0,61 \mathrm{BC}$ & $57,26 \pm$ & $0,61 \mathrm{D}$ & $57,42 \pm$ & $0,61 \mathrm{D}$ & $58,77 \pm$ & $0,61 \mathrm{CD}$ & $58,61 \pm$ & $0,61 \mathrm{CD}$ & $\begin{array}{c}60,09 \pm \\
3,50\end{array}$ \\
\hline $\mathrm{CV}(\%) \mathrm{JAR}$ & 0 & & & & 1, & & & & & & & & & & 5,82 \\
\hline \multicolumn{5}{|c|}{$\begin{array}{l}\text { Grande moyenne Portulaca sp. } \\
\text { Grande moyenne Cyperus rotundus } \\
\text { Grande moyenne traitement }\end{array}$} & \multicolumn{11}{|c|}{$\begin{array}{c}62,57 \pm 0,33 a \\
57,62 \pm 0,33 b \\
60,09 \pm 3,50\end{array}$} \\
\hline
\end{tabular}

\begin{tabular}{|c|c|c|c|c|c|}
\hline Source de variation & Traitement & Espèce & JAR & Traitement * JAR & Traitement $*$ Espèce \\
\hline Ddl & 3 & 1 & 6 & 18 & 3 \\
\hline Probabilité (P) & $0,000^{* * *}$ & $0,000^{* * *}$ & $0,000^{* * *}$ & $0,000^{* * *}$ & $0,000^{* * *}$ \\
\hline $\mathbf{F}$ & 852,34 & 114,11 & 20,84 & 10,91 & 22,52 \\
\hline
\end{tabular}

C : Cyperus rotundus; P : Portulaca oleracea et Portulaca quadrifida à la fois

Les valeurs moyennes suivies des différentes lettres a e tb présentent des différences très hautement significatives au seuil de signification de $5 \%$ d'après le test de Tukey pour la colonne considérée

Les lettres A, B, C et D indiquent une différence significative entre les moyennes des traitements au seuil de 5\% aux différentes périodes végétatives de l'oignon

$* * *: \mathrm{p}=0,000<0,001$ signifie très hautement significatif 
Tableau 4 : Biomasses spécifiques et totales ( $\mathrm{kg} / \mathrm{ha}$ de matière sèche) des mauvaises herbes et synthèse des résultats statistiques liées aux différentes méthodes de lutte

\begin{tabular}{l|c|c|c|c}
\hline \multirow{2}{*}{\multicolumn{1}{c}{ Traitements }} & \multicolumn{4}{c}{ Biomasse (kg/ha de matière sèche) } \\
\cline { 2 - 5 } & $\begin{array}{c}\text { Portulaca oleracea et Portulaca } \\
\text { quadrifida }\end{array}$ & $\begin{array}{c}\text { Cyperus } \\
\text { rotundus }\end{array}$ & $\begin{array}{c}\text { Autres } \\
\text { espèces }\end{array}$ & Totale \\
\hline Sarclo-binages répétés sur & & & & \\
\hline Faiblement envahie (PFaE) & $90 \mathrm{~d}$ & 410c & $130 \mathrm{c}$ & $630 \mathrm{c}$ \\
\hline Fortement envahie (PFoE) & $1200 \mathrm{a}$ & $1200 \mathrm{a}$ & $330 \mathrm{a}$ & $2720 \mathrm{a}$ \\
\hline
\end{tabular}

Application d'herbicide sur parcelle fortement envahie (densité de mauvaises herbes $>$ à 4 plants $/ \mathrm{m}^{2}$ ) combinée aux sarclobinages répétés

\begin{tabular}{l|c|c|c|c}
\hline Round Up (Round Up) & 360c & 530c & $100 \mathrm{c}$ & $980 \mathrm{~b}$ \\
\hline Agristomp (Stomp) & $560 \mathrm{~b}$ & $850 \mathrm{~b}$ & $200 \mathrm{~b}$ & $600 \mathrm{c}$ \\
\hline Source de variation & & & & \\
\hline Moyenne & $\mathbf{0 , 8 5}$ & $\mathbf{1 , 1 5}$ & $\mathbf{0 , 2 9}$ & $\mathbf{1 , 9 9}$ \\
\hline dd1 & $\mathbf{2 2}$ & 22 & 22 & 22 \\
\hline Probabilité $(\boldsymbol{P}<\boldsymbol{F})$ & $\mathbf{0 , 0 0 6 * *}$ & $\mathbf{0 , 0 0 4 * *}$ & $\mathbf{0 , 0 0 2 * *}$ & $\mathbf{0 , 0 0 0 * * *}$ \\
\hline
\end{tabular}

Les valeurs moyennes suivies des différentes lettres $a, b$, c et d sont significativement différentes au seuil de signification de $5 \%$, d’après le test de Tukey pour la colonne considérée

***: Très hautement significatif ; ** Très significatif ; *: significatif

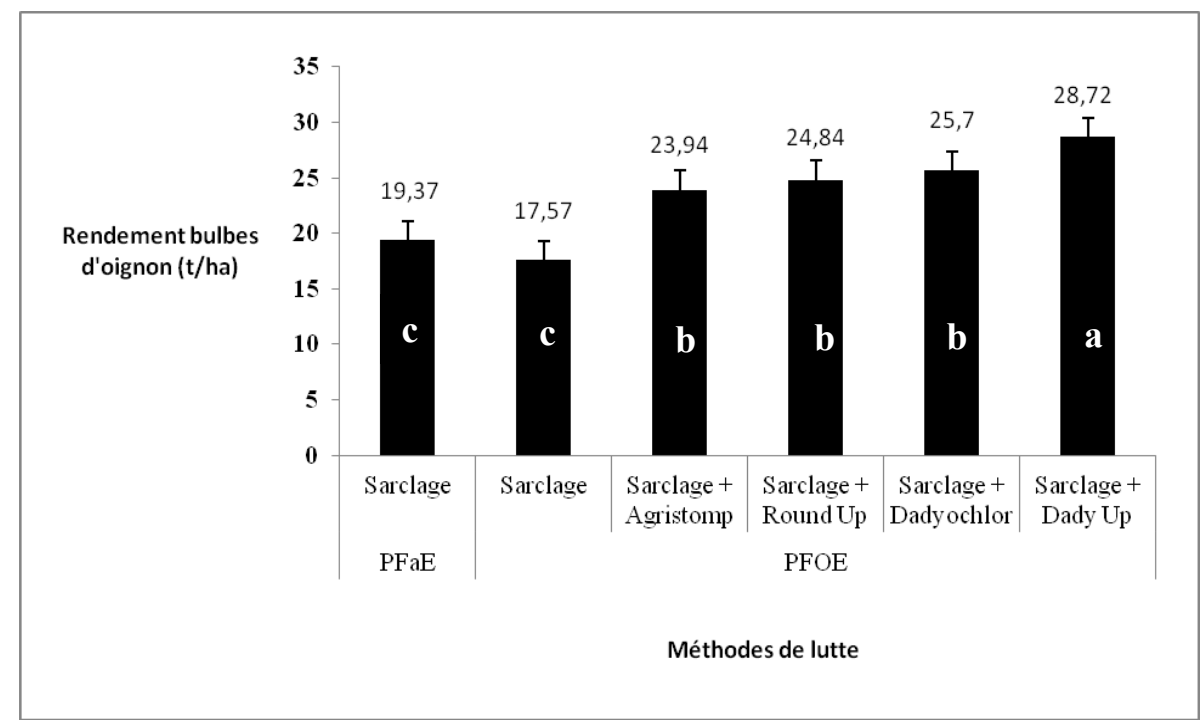

Figure 4 : Rendements en bulbes d'oignon (t/ha) obtenus avec les différentes méthodes de lutte testées

Légende : PFoE : Parcelle fortement envahie, PFaE : Parcelle faiblement envahie, $\mathrm{T}:$ Ecart type. Les nombres portant les différentes lettres $(a, b$ et $c)$ présentent des différences très hautement significatives au seuil de signification de $5 \%$ d'après le test de Tukey.

Tous les traitements herbicides ont engendré des niveaux de rendement compris entre 24 t/ha et $28,72 \mathrm{t} / \mathrm{ha}$. Ces valeurs étaient significativement plus élevées que celles des pratiques paysannes de sarclo-binages exclusifs effectués sur des parcelles faiblement enherbées (PFaE) et fortement enherbées (PFoE) qui ont engendré un niveau de rendement 
statistiquement identique, compris entre 17,57 t/ha et 19,37 t/ha. L'herbicide le plus efficace est le Dady Up avec un rendement de 28,72 t/ha qui était significativement le plus élevé. C'est la combinaison des sarclo-binages avec l'application de l'herbicide Dady Up qui a procuré le gain de rendement le plus élevé, représentant $48,27 \%$ par rapport à la pratique paysanne des sarclo-binages sur parcelles fortement ou faiblement envahies. Elle est suivie de la combinaison des sarclo-binages avec l'application des herbicides Dadyochlor, Round Up et Agristomp qui ont engendré un gain statistiquement identique, compris entre $23,59 \%$ et $32,68 \%$ (tableau 5 ).

Tableau 5 : Evaluation de l'effet synergique de la combinaison des pratiques paysannes de sarclobinages et d'application d'herbicides

\begin{tabular}{|c|c|c|c|}
\hline \multirow[t]{2}{*}{ Comparaison des traitements } & \multirow{2}{*}{$\begin{array}{c}\text { Rendement en } \\
\text { bulbes d'oignon } \\
(\mathrm{t} / \mathrm{ha})\end{array}$} & \multicolumn{2}{|c|}{$\begin{array}{l}\text { Différence et signification pat } \\
\text { rapport à PFoE/PFaE }\end{array}$} \\
\hline & & t/ha & $\%$ \\
\hline \multicolumn{4}{|l|}{ Sarclo-binages répétés sur parcelle } \\
\hline Faiblement envahie (PFaE) & $17,57 \mathrm{c}$ & Oc & $0 \mathrm{c}$ \\
\hline Fortement envahie (PFoE) & $19,37 \mathrm{c}$ & Oc & Oc \\
\hline \multicolumn{4}{|c|}{ Sarclo-binages répétés sur parcelle fortement envahie combinés à : } \\
\hline Agristomp (Stomp) & $23,94 \mathrm{~b}$ & $4,57 b$ & $23,59 \mathrm{~b}$ \\
\hline Round Up (Round Up) & $24,84 \mathrm{~b}$ & $5,47 \mathrm{~b}$ & $28,24 \mathrm{~b}$ \\
\hline Dadyochlor (Dadyochlor) & $25,70 \mathrm{~b}$ & $6,33 \mathrm{~b}$ & $32,68 \mathrm{~b}$ \\
\hline Dady Up (Dady Up) & $28,72 \mathrm{a}$ & $9,35 \mathrm{a}$ & $48,27 \mathrm{a}$ \\
\hline
\end{tabular}

Les nombres portant les différentes lettres $a, b$ et $c$ présentent des différences très hautement significatives au seuil de signification de $1 \%$, d'après le test de Tukey.

4.5 Rentabilité économique des sarclobinages répétés et de leur combinaison avec l'application d'herbicide: Les paramètres utilisés pour apprécier la rentabilité économique étaient le produit brut, la marge brute, le revenu partiel et le ratio produit brut sur charges totales (Midingoyi, 1997a ; Midingoyi, 1997b ; Alimi et Manyong, 2000; Coulibaly et N'kamleu, 2007). Les coûts de main d'œuvre d'application d'herbicide et de sarclages sont calculés respectivement sur la base de $2000 \mathrm{~F}$ CFA et de 1000 F CFA, l'équivalent de la rémunération d'un actif par jour (h.j). Trois sarclages renforcés par l'arrachage manuel et l'enfouissement de la biomasse sarclée sont considérés pour chaque méthode de lutte, pour une quantité totale de main d'œuvre évaluée à 39 h.j. Les coûts d'achat des doses d'herbicides sont calculés sur la base de $6 \mathrm{l} /$ ha pour le Round Up, de 8 1/ha pour le Dady Up, de 2,5 1/ha pour l'Agristomp et de 1,5 1 /ha pour le Dadyochlor. Le coût d'achat de Round Up était de 5000 F CFA/1, celui de Dady Up de 6000 F CFA/1, Agristomp a couté 7500 F CFA/1 et le Dadyochlor a coûté 9500 F CFA/l. L'estimation des paramètres de rentabilité économique des différentes méthodes de lutte sur 1 ha est présentée au tableau 6. Le classement par ordre décroissant des valeurs du rendement, du produit brut, de la marge brute, $\mathrm{du}$ revenu partiel et $\mathrm{du}$ ratio produit brut/charges totales des méthodes de lutte a donné Dady Up, Dadyochlor, Round Up, Agristomp et les sarclages répétitifs (pratique paysanne) dans cet ordre respectivement $1^{\text {er }}$, $2^{\text {ème }}, 3^{\text {ème }}, 4^{\text {ème }}$ et $5^{\text {ème }}$. Tous les paramètres économiques des pratiques d'application d'herbicide étaient supérieurs à ceux de la pratique paysanne. Ainsi donc, les gains de rendement engendrés par l'effet synergique de 
la combinaison des pratiques chimique et l'achat et à la main d'œuvre d'application des culturale ont compensé les surcoûts liés à traitements herbicides.

Tableau 6 : Rentabilité économique en F CFA et en pourcentage relatif de la pratique paysanne (\% entre parenthèses) des méthodes de lutte testées sur 1 ha d'oignon

\begin{tabular}{|c|c|c|c|c|c|}
\hline \multirow{2}{*}{ Paramètres économiques } & \multicolumn{2}{|c|}{$\begin{array}{l}\text { Pratique paysanne } \\
\text { combinée à }\end{array}$} & \multicolumn{2}{|c|}{$\begin{array}{l}\text { Pratique paysanne } \\
\text { combinée à }\end{array}$} & \multirow{2}{*}{$\begin{array}{l}\text { Pratique } \\
\text { paysanne } \\
\text { exclusive }\end{array}$} \\
\hline & Dady Up & Dadyochlor & Round up & Agristomp & \\
\hline Rendement (R) en $\mathrm{kg} / \mathrm{ha}$ & $\begin{array}{c}28.720 \\
(163,46)\end{array}$ & $\begin{array}{c}25.700 \\
(146,27)\end{array}$ & $\begin{array}{l}24.800 \\
141,15\end{array}$ & $\begin{array}{c}23.940 \\
(136,25)\end{array}$ & $\begin{array}{c}17.570 \\
(100)\end{array}$ \\
\hline Prix du kg d'oignon (FCFA) & 175 & 175 & 175 & 175 & 175 \\
\hline $\begin{array}{l}\text { Coût d'achat des herbicides } \\
\text { (FCFA) }\end{array}$ & 48.000 & 14.250 & 30.000 & 18.750 & 0 \\
\hline $\begin{array}{l}\text { Coût d'application des } \\
\text { herbicides (FCFA) }\end{array}$ & 2.000 & 2.000 & 2.000 & 2.000 & 0 \\
\hline Coût des sarclages (FCFA) & 39.000 & 39.000 & 39.000 & 39.000 & 39.000 \\
\hline $\begin{array}{l}\text { Coûts Variables Partiels (CVP) } \\
\text { en FCFA }\end{array}$ & 89.000 & 55.250 & 71.000 & 59.750 & 39.000 \\
\hline Charges totales (CT) & 771.500 & 737.750 & 753.500 & 742.250 & 721.500 \\
\hline $\begin{array}{l}\text { Produit brut }(\mathrm{PB})= \\
\text { Rendement }(\mathrm{R}) * \text { Prix en FCFA }\end{array}$ & $\begin{array}{c}5.026 .000 \\
(163,46)\end{array}$ & $\begin{array}{c}4.497 .500 \\
(146,27)\end{array}$ & $\begin{array}{c}4.340 .000 \\
(141,15)\end{array}$ & $\begin{array}{c}4.189 .500 \\
(136,25)\end{array}$ & $\begin{array}{l}3.074 .750 \\
\quad(100)\end{array}$ \\
\hline $\begin{array}{l}\text { Marge Brute }(\mathrm{MB})= \\
\mathrm{PB}-\mathrm{CT} \text { en FCFA }\end{array}$ & $\begin{array}{l}4.254 .500 \\
(180,79)\end{array}$ & $\begin{array}{l}3.759 .750 \\
(159,77)\end{array}$ & $\begin{array}{c}3.586 .500 \\
(152,41)\end{array}$ & $\begin{array}{l}3.447 .250 \\
(146,49)\end{array}$ & $\begin{array}{l}2.353 .250 \\
(100)\end{array}$ \\
\hline $\begin{array}{l}\text { Ratio Produit brut / Charges } \\
\text { totales }\end{array}$ & $\begin{array}{c}6,51 \\
(152,82)\end{array}$ & $\begin{array}{c}6,10 \\
(143,19\end{array}$ & $\begin{array}{c}5,76 \\
(135,21)\end{array}$ & $\begin{array}{c}5,64 \\
(132,39)\end{array}$ & $\begin{array}{c}4,26 \\
(100)\end{array}$ \\
\hline $\begin{array}{l}\text { Revenu Partiel (RP) = Produit } \\
\text { brut-CVP (FCFA) }\end{array}$ & $\begin{array}{l}4.937 .000 \\
(162,63)\end{array}$ & $\begin{array}{c}4.442 .250 \\
(146,33)\end{array}$ & $\begin{array}{c}4.269 .000 \\
(140,62)\end{array}$ & $\begin{array}{c}4.129 .750 \\
(136,04)\end{array}$ & $\begin{array}{c}3.035 .750 \\
(100)\end{array}$ \\
\hline
\end{tabular}

\subsection{Appréciation paysanne et devenir} des résultats : De l'avis des producteurs, les herbicides Round Up et Dady Up ont exprimé une efficacité sur Cyperus rotundus en culture d'oignon déjà à partir du $5^{\text {ème }}$ jour suivant l'application effectuée avant le labour. Les producteurs une fois convaincus des résultats de l'essai, étaient préoccupés de mieux gérer l'enherbement de l'oignon par la mise en pratique à petite échelle de la lutte chimique contre les mauvaises herbes de l'oignon et du riz. C'est ainsi que l'utilisation d'Agristomp à la dose de 2,75 1/ha par une vingtaine de

\section{DISCUSSION}

5.1 Phytotoxicité des herbicides : Les niveaux de phytotoxicité de $7 \%$ et de $3 \%$ évalués au $15^{\text {ème }}$ JAR respectivement pour les herbicides Round Up et l'Agristomp étaient dus producteurs est observée. Les producteurs ont adapté son utilisation en l'appliquant 24 à 48 heures après le repiquage et non à la préirrigation tel qu'expérimenté dans le cadre de la présente étude. Des applications d'herbicides totaux à base de glyphosate pour l'installation du maïs, du riz, du mil et du sorgho qui sont des précédents culturaux de l'oignon sont aussi signalées. D'après ces constatations, une large adoption à long terme de ces herbicides notamment Agristomp pour une meilleure gestion de l'enherbement de l'oignon était envisageable.

à leurs caractères total et sélectif respectifs. Pour l'herbicide Round Up, sa phytotoxicité relativement élevée est attribuée à la texture sablo-limoneuse ou sablo-argileuse des sols en 
rapport avec un niveau de dégradation de la fertilité. Ce résultat justifiait l'agrément d'homologation et l'autorisation provisoire de vente du glyphosate aux teneurs de $360 \mathrm{~g} / 1$ et de $700 \mathrm{~g} / 1$ au Bénin (CNAC, 2014). En effet, un risque de phytotoxicité des herbicides à pénétration foliaire à base de matières actives telles que le glyphosate, qui sont inactivées au contact du sol, est surtout observé dans les sols dégradés très sableux, sans matière organique, ni argile (Anonyme, 2002).

5.2 Effet des pratiques de lutte sur le recouvrement des mauvaises herbes: La meilleure maittrise des mauvaises herbes obtenue avec les herbicides traduisait un certain intérêt de la lutte chimique. Ee couple Portulaca oleracea et Portulaca quadrifida était plus abondant et plus dominant que Cyperus rotundus. Toutefois, Cyperus rotundus est mieux contrôlé par Round Up que par Agristomp, tandis que Portulaca oleracea et Portulaca quadrifida étaient mieux contrôlés par Agristomp. L'efficacité des herbicides comparativement à celle des sarclages manuels répétitifs réalisés de façon exclusive peut être justifiée par les avis de N'diaye et al. (2009a), de N'diaye et al. (2009b) et de N'diaye et al. (2009c) selon lesquels " Cyperus rotundus est difficile à éliminer par le sarclage manuel dans les parcelles de maraîchage, du fait de son mode de reproduction presque exclusivement végétatif ». La faible maitrise de Portulaca oleracea et de Portulaca quadrifida par les sarclages manuels pouvait s'expliquer, d'après les résultats de la présente étude, par leur capacité à survivre aux conditions d'humidité liées à l'arrosage. Cette constatation était similaire à celle de Fall et al. (1978) qui ont observé au Sénégal, l'émergence de nouvelles pousses de Commelina benghalensis lorsqu'une pluie survenait 8 jours après le sarclage. Ce résultat est approfondi par Gounifio (1988) qui a émis l'hypothèse qu'« en conditions d'humidité, la maitrise des infestations de cette espèce par le sarclage devient difficile ». La difficulté de maîtriser ces espèces relevait d'une préoccupation qui n'est guère résolue depuis les années 1990 dans bon nombre de systèmes culturaux. Au Bénin, la maitrise difficile des Commelinacées en culture cotonnière avec des herbicides spécifiques est évoquée (RCF, 1994). En effet, le temps de travail requis pour le sarclage et l'extension des superficies des parcelles infestées par cette espèce dans le Nord-Bénin est rapporté par Ahanchédé (1995).

\subsection{Effet des pratiques de lutte sur la} biomasse des mauvaises herbes: La meilleure efficacité obtenue pour les deux herbicides totaux, comparativement aux deux herbicides sélectifs et aux pratiques paysannes, pouvait s'expliquer par le fait que leur application aux deux doses, est réalisée sur les mauvaises herbes au stade plantules sensibles (Anonyme, 2002). Cette constatation confirmait la difficulté de maîtriser Portulaca quadrifida, Portulaca oleracea et Cyperus rotundus dans un contexte écologique où les sarclo-binages, l'irrigation et la fumure minérale intense étaient des facteurs favorables à l'enherbement (Bello et al. (2012a); Bello, 2013). En effet, les interventions de désherbage devaient tenir compte des contraintes de l'itinéraire technique de la culture et l'utilisation des produits systémiques comme le glyphosate en cas d'infestation par des espèces vivaces comme Cyperus rotundus devait être envisagée (Anonyme, 2002). La pratique exclusive des sarclo-binages à la houe et à la binette entraînait la fragmentation et la dispersion des propagules de ces mauvaises herbes, ce qui facilitait leur multiplication et par conséquent, un recouvrement intense qui rendait difficile leur contrôle tel que rapporté par Dembélé et al. (1996) ainsi que par N'diaye et al. (1997).

Les herbicides Round Up et Agristomp étaient plus efficaces sur les Portulacacées que sur la Cypéracée, le premier mieux que le deuxième. L'effet dépressif des herbicides sur les mauvaises herbes pouvait être lié à un ralentissement de l'activité physiologique des 
espèces, notamment à partir du stade juvénile. Ces résultats traduisaient l'efficacité des herbicides à partir du stage juvénile des plantules et présageaient de l'opportunité de démarrer les sarclo-binages à partir de la troisième semaine après le repiquage.

\subsection{Effet des pratiques de lutte sur le rendement en bulbes d'oignon :} L'application d'herbicides suivie de sarclobinages a engendré un gain de rendement comparativement aux sarclo-binages exclusifs, respectivement de 48,27\% pour Dady Up, et de $23,59 \%$ à 32,68\% pour Dadyochlor, Agristomp et Round Up, traduisant un effet synergique de l'usage combiné des deux méthodes. Ce résultat a confirmé la recommandation de Leblanc (2008) qui a suggéré le désherbage mécanique des inter-rangs en début de saison pour renforcer l'efficacité des herbicides. Ces résultats ont confirmé ceux de Mirza et al. (2007) qui ont observé que le rendement en grain du riz était passé de $2,4 \mathrm{t} / \mathrm{ha}$ à $5,2 \mathrm{t} / \mathrm{ha}$ avec l'application d'herbicide à base de pretilachlor, suivi d'un désherbage manuel contre 2,4 t/ha sans une application d'herbicide. Les niveaux de rendement en bulbes d'oignon obtenus pour la variété locale avec l'application des différents herbicides, compris entre $23,94 \mathrm{t} /$ ha et $28,72 \mathrm{t} /$ ha, étaient comparables aux 29,9 t/ha obtenus avec la même variété par Assogba-Komlan et al. (2007) au Sud-Bénin et aux valeurs comprises entre $26,20 \mathrm{t} / \mathrm{ha}$ et $33,8 \mathrm{t} / \mathrm{ha}$ obtenus par Bello et al. (2004), puis par Bello et al. (2012a, 2012b) au Nord-Bénin dans diverses conditions de sarclobinages, de fumure et de densité optimales. Les faibles rendements obtenus avec la pratique traditionnelle des sarclages exclusifs traduisaient en fait un retard de croissance lié à un effet de compétition des mauvaises herbes, tel que rapporté par Deat $(1973$; 1986) aux premiers stades de développement du cotonnier et qui a suggéré d'y remédier par une application d'herbicide.
Les résultats de la présente étude corroborent aussi les recommandations de Dembélé et al. (1996), puis de N'diaye et al. (1997) relatives à l'utilisation du glyphosate pour une élimination presque totale de Cyperus rotundus. En considérant cet intérêt d'une meilleure maittrise de l'enherbement au moyen d'herbicides, Akobundu (1987) avait donc raison de soutenir que la lutte chimique devait être encouragé dans le futur dans les pays en développement afin d'améliorer les conditions de réalisation du désherbage. La preuve manifeste était l'utilisation de nos jours, de l'herbicide Agristomp en milieu paysan, contrairement à l'utilisation limitée au Mali, de la technique d'inondation des parcelles, de la culture de la patate douce, du labour de fin de cycle et notamment de la pratique de sarclages répétés, à cause de leurs coûts élevés et de leur inefficacité par rapport à la réduction des pertes de rendement causées par Cyperus rotundus qui étaient les raisons avancées par les producteurs (N'diaye et al., 1997). L'utilisation alternée des herbicides totaux Dady Up et Round Up et des herbicides sélectifs Dadyochlor et Agristomp devait permettre de prévenir la sélection, voire la résistance à long terme de certaines espèces de mauvaises herbes, telle que suggéré par Akobundu (1987) ainsi que par Hugh et al. (2001).

5.6 Rentabilité économique des méthodes de lutte: En situation de sarclobinages exclusifs, les niveaux de rendement de l'oignon obtenus étaient inférieurs à ceux attendus, en raison de l'effet dépressif des rendements du fait des interactions négatives des mauvaises herbes sur les cultures. Deat (1973) et Deat (1986) indiquaient que les premiers stades de développement des cultures correspondaient au retard de croissance lié à l'effet de compétition des mauvaises herbes qui se traduisait par une dépréciation de la qualité du produit et une baisse des rendements. Les pertes de rendement engendrées variaient de $35 \%$ à 90\% selon les cultures (Marnotte, 1995). 
Les observations directes effectuées sur le terrain ont montré qu'une application d'herbicide sur un hectare durait en moyenne une journée de travail contre 13 jours pour un désherbage manuel. Ce résultat était similaire à celui de Marnotte (1995) qui a rapporté que « l'application d'herbicide sur un hectare requiert une journée de travail contre 10 à 20 jours pour le sarclage manuel selon le stade de la culture et des mauvaises herbes ». L'application d'herbicide permet une augmentation de la production, appréciable par rapport aux pratiques traditionnelles et le gain de temps et de main d'œuvre permis par cette technique est particulièrement élevé (Deat, 1973 ; Deat, 1986).

\section{CONCLUSION}

Les herbicides sélectifs Agristomp ou Dadyochlor et surtout les totaux à base de glyphosate comme Dady Up sont efficaces pour renforcer l'efficacité de la méthode culturale des sarclo-binages. L'effet synergique induit et la possibilité de sédentariser la culture sont des atouts de la combinaison. L'utilisation alternée dans le temps, des herbicides sélectifs
Les résultats économiques obtenus corroborent ceux de Martin et Gaudard (1996) qui ont expliqué que les traitements à base d'herbicides de prélevée préconisés en région cotonnière au Nord-Cameroun étaient rentables grâce aux gains de temps de travail et de production obtenus, tout en précisant que le glyphosate était utilisable en traitement de pré-semis et que son mode d'application était plus contraignant. L'engouement des producteurs à l'adoption de Agristomp confirme la remarque de Marnotte (1995) qui avait évoqué qu'« une application d'herbicide implique toutefois des conditions économiques favorables ainsi qu'une bonne formation du personnel d'encadrement et des agriculteurs ».

et totaux permet de minimiser les risques sur l'environnement et de prévenir la résistance de certaines espèces de la flore. Cette attitude suscite la préoccupation de rechercher une meilleure stratégie de gestion des mauvaises herbes qui concilie à la fois efficacité, viabilité, durabilité et rentabilité.

\section{REFERENCES BIBLIOGRAPHIQUES}

Aboué M : 2005. Législation et réglementation phytosanitaires et leurs applications : Homologation interafricaine (HIP). In : Adégnika M., Okry F. et Ouédraogo B., éds. Les évolutions en cours dans les filières cotonnières : conséquences pour la Recherche agronomique et l'acquisition des intrants par les agriculteurs. Actes de la conférence Coton de l'Afrique de l'Ouest et du Centre, 10-12 mai 2005, Cotonou, Bénin : INRAB, IFDC et CORAF/WECARD, 64-76.

Ahanchédé A: 1995. Quelques aspects de la nuisibilité de Commelina benghalensis L. et de C. bracteosa L. sur le maïs. Bulletin de la Recherche Agronomique (BRAB) $11: 1-7$.
Akobundu I.O: 1987. Weed science in the Tropics. Principles and practices. ISBN 047191544 0. Wiley J. and Sons Ltd. (éds), Chichester.

Alimi T. and Manyong VM: 2000. Partial budget analysis for on farm research. Research guide $\mathrm{n}^{\circ}$ 65. International Institue of Tropical agriculture (IITA), Ibadan, Nigeria.

Anonyme: 1995. Index phytosanitaire. ACTA (Association de coopération technique agricole), éd., Paris, France.

Anonyme: 2002. Mémento de l'agronome, éditions 2002. CTA, CIRAD et GRET (éds), Version CD-ROM.

Anonyme: 2006b. Notice d'utilisation de l'herbicide Dadyochlor: Etiquette de 
conditionnement, AGRIMAT, SDI, Cotonou, Bénin.

Anonyme: 2008a. Notices de produits herbicides à base de glyphosate : Etiquette de conditionnement, Razer Int (éd), SDI, Cotonou, Bénin.

Anonyme: 2008b. Service Questions et Réponses (SQR) Bénin: Pourquoi manger le souchet ? Série "Accès au Marché Local ». ONASA, CTA (éds). Cotonou, Bénin.

Anonyme: 2006a. Notice d'utilisation de l'herbicide Agristomp 500 E: Etiquette de conditionnement AGRIMAT, SDI, Cotonou, Bénin.

Assogba-Komlan F., Sikirou R., Singbo A.G. et Azagba J : 2006. Mieux conduire la culture de l'oignon sous régime pluvial au Sud du Bénin. Igué D., Affonon E. et Akpadji J., (éds.). Référentiel Technico-économique, ISSN : 18405479, ISBN : 99919-60-79-1, République du Bénin.

Bello S: 2012. Analyse diagnostique de la production et de la commercialisation de l'oignon de 1995 à 2009 au Nord-Est $\mathrm{du}$ Bénin. Bulletin de la Recherche Agronomique du Bénin (BRAB) 71 : 46-61.

Bello S : 2013. Développement participatif d'un itinéraire technique de gestion intégrée des mauvaises herbes de l'oignon (Allium repa L.) au Nord-Est du Bénin. Thèse de doctorat unique, Université d'Abomey-Calavi, Faculté des Sciences Agronomiques, Abomey-Calavi (Bénin), $352 \mathrm{p}$.

Bello S., Ahanchédé A., Amadji G., Gbèhounou G. et Aho N : 2012b. Effet de la fumure minérale sur l'enherbement et la production de l'oignon (Allium cepa L.) au Nord-Est du Bénin. Int. J. Biol. Chem. Sci. 6(6), 40584070.

Bello S., Ahanchédé A., Amadji G., Gbèhounou G.et Aho N : 2013.
Evolution saisonnière de la flore sarclée des parcelles d'oignon (Allium cepa L.) au Nord-Est du Bénin. Communication soumise pour participation et publication aux actes de la $22^{\text {ème }}$ Conférence du COLUMA organisée par l'Association Française de la Protection des Plantes (AFPP) : Journées internationales sur la lutte contre les mauvaises herbes, prévues à Dijon les 10, 11 et 12 décembre 2013. 10 p.

Bello S., Ahanchédé A., Gbèhounou G., Amadji G. et Aho N. 2013a. Diversité floristique, ethnobotanique et taxonomie locale des mauvaises herbes de l'oignon au Nord-Est du Bénin. TROPICULTURA, 2013, 31 (2) : 53-63. ISSN 0771-3312. Site web : http://www.tropicultura.org/

Bello S., Amadji G., Gbèhounou G., Aho N. et Ahanchédé A : 2012a. Influence de la densité de repiquage sur l'enherbement et la production de l'oignon (Allium cepa L.) au Nord-est du Bénin. J. Anim. Plant Sci., 2012, 15(1) 2074-2082. ISSN 20717024. Site web: http://www.m.elewa.org/JAPS.

Bello S., Assogba-Komlan F.V. et Baco M.N: 2004. Guide pratique pour la production de bulbes d'oignon dans le département de l'Alibori. Igué D., Affonon E. et Akpadji J., (éds.). Référentiel Technico-économique, INRAB/AFD/PADSE, ISBN $\mathrm{N}^{\circ}$ 99919-51-64-4, République du Bénin, $52 \mathrm{p}$.

CNAC (Comité National d'Agrément et de Contrôle des Produits Phytopharmaceutiques), 2012. Liste des produits phytopharmaceutiques sous autorisation provisoire de vente (APV) et agrement homologation ( $\mathrm{AH})$ : Liste actualisée en janvier 2012. DPV/MAEP, Porto-Novo, Bénin. 
CNAC (Comité National d'Agrément et de Contrôle des Produits Phytopharmaceutiques), 2014. Liste des produits phytopharmaceutiques sous autorisation provisoire de vente (APV) et agrement d'homologation $(\mathrm{AH})$. Liste actualisée en Juin 2014. DPV/MAEP, Porto Novo, Bénin.

Coulibaly O. et N'kamleu B. 2007. Manuel de formation sur les modèles d'analyse économétriques pour économistes agricoles. Institut International d'Agriculture Tropicale (IITA), Laboratoire d'Analyse et de Renforcement de Capacités en Sciences Sociales. Traduit de l'anglais en français par Théodore Nouhoheflin, $28 \mathrm{p}$.

Deat M: 1973. Etude économique de deux herbicides en culture cotonnière en Côte d'Ivoire. Coton et fibres tropicales 28(2) : 293-295.

Deat M : 1986. Le désherbage des cultures, un facteur d'optimisation des rendements. In : CIRAD-IRCT Montpellier (éd). IV $^{\text {ème }}$ congrès sur la protection de la santé humaine et des cultures en milieu tropical, 2-4 juillet 1986, Marseille, France : Tiré à part, 10 p.

Dembélé D., N'diaye M. et Traoré N : 1996. Maitrise des mauvaises herbes des cultures maraîchères par la lutte chimique : expérimentation sur le glyphosate et l'oxadiazon. Station de Recherche Agronomique, Baguinéda, Mali. Bulletin de liaison 10(31) : 64-67.

Fall M., Herdandez S., Pirot R., Pocthier G. et Tchakerian E: 1978. Utilisation des herbicides au Sénégal : principales contraintes et possibilités d'introduction en milieu paysan. III ${ }^{\text {ème }}$ symposium sur le désherbage des cultures tropicales. 17 au 21 Septembre 1978, Dakar, 31-45.

Gnanglè C., Bello S. et Ogodja J.O : 2002. Diagnostic participatif du village de Tomboutou dans la commune de
Malanville. Rapport technique, RD Alibori/CRAN/INRAB, Kandi, Bénin.

Gotoéchan-Hodonou H. et Savi A.D. 2001.

Etude diagnostique sur la production de l'oignon dans le département de l'Alibori. In :

INRAB/GTZ/Coopération

Néerlandaise, éds. Actes de l'Atelier Scientifique Nord, Edition 2001. Parakou, Bénin.109-119. ISBN : 99919913-2-8.

Gounifio P. 1988. Biomorphologie de Commelina benghalensis $L$. pour une meilleure approche de la lutte raisonnée. Thèse de doctorat, Université des Sciences et Techniques du Languedoc, Montpellier (France).

Hugh M., Tardif F. et Ferguson G: 2001. La résistance des mauvaises herbes aux herbicides. Fiche technique. Ministère de l'Agriculture, de l'Alimentation et des Affaires Rurales (MAAARO), http://www.gov.on.ca/MBS/french/co mmon/queens.html., Consulté le 19 septembre 2010 à $22 \mathrm{~h} 05 \mathrm{mn}$.

INRAB : 2005. Rapport d'assises du Comité Régional de Recherche et de Développement (CRRD) du Centre et du Nord ; MAEP/INRAB, Bénin.

Marnotte P: 1995. Utilisation des herbicides : contraintes et perspectives. Agriculture et développement, $\mathrm{n}^{\circ} 7$, septembre $1995: 12$ 21.

Marnotte P : 2000. La gestion de l'enherbement et l'emploi des herbicides dans les systèmes de culture en zone soudanosahélienne en Afrique de l'Ouest et du Centre. Formation du CIRAD, 2000. CIRAD-CA-G.E.C. -AMATROP, France.

Marnotte P, Perret D et Carrara A : 2004. Enquête sur l'enherbement des rizières de Camargue (France). In : AFPP, éd., $12^{\text {ème }}$ Colloque international sur la 
biologie des mauvaises herbes. 2004. Dijon : France.

Marnotte P. et Tehia K.E: 1991. Les herbicides dans les cultures vivrières en Côte d'Ivoire : les produits vulgarisables. Sabel PV Info 37: 17-23.

Martin J et Gaudard L : 1996. Paraquat, diuron et atrazine pour renouveler le désherbage chimique au NordCameroun. Agriculture et développement, $\mathrm{n}^{\circ} 11$, septembre 1996: 53-67.

Marty J.P., Perny R.A. et Hilaire A : 1977. Evolution de la flore adventice en cultures de maïs et de sorgho appartenant à différentes rotations irriguées ou non. Note présentée par M. S. Hénin. Compte rendu de l'Académie d'Agriculture de France, Année 1977, $\mathrm{N}^{\circ} 4$.

Midingoyi S : 1997a. Concepts et méthodes d'évaluation économique des essais et des tests en milieu réel. Cours de formation des équipes R-D. Guide de Recherche $\mathrm{n}^{\circ} 2$, INRAB/LESR, PortoNovo, Bénin.

Midingoyi S : 1997b. Méthodes de collecte de données socio-économiques en recherche-développement. Cours de formation des équipes R-D, PortoNovo, mai 1997. INRAB/LESR, Bénin.

Mirza H., Kamrun N. et Karim RMd : 2007. Effectiveness of different weed control methods on the performance of transplanted rice. Weed Science Society of Pakistan, 2007. Pak J. Weed Sci. Res. 13(1-2) : 17-25.

N'diaye M., Traoré N. et Dembélé D : 1997. Etude de l'effet du piochage répété des parcelles, de la culture de patate douce et de l'utilisation du Round Up dans la lutte contre Cyperus rotundus en parcelles de maraîchage. Station de Recherche Agronomique, Baguinéda, Mali. Bulletin de Liaison 12-30 novembre 1997 : 37-41.

N'Diaye M., Dembélé B. et Diop O : 2009c. Mauvaises herbes des cultures maraîchères : Fréquence et abondance dans les parcelles de trois secteurs de production de la zone périurbaine de Bamako. Les cabiers de l'économie rurale 8 : 37-46.

N'Diaye M., Dembélé M. et Traoré N : 2009b. Maîtrise des mauvaises herbes des cultures maraichères par la lutte par la lutte chimique : expérimentation sur le Glyphosate et l'oxadiazon. Institut d'économie rurale (IER). Les cabiers de l'économie rurale $8: 31-36$.

N'Diaye M., Traoré N. et Dembélé D : 2009a. Étude de l'effet du piochage répété des parcelles, de la culture de patate douce et de l'utilisation du Roundup dans la lutte contre Cyperus rotundus en parcelles de maraîchage. Institut d'économie rurale (IER). Les cahiers de l'économie rurale $8: 25-30$

RCF : 1994. Rapport de synthèse pour la campagne 1993. Unité de recherches Coton et Fibres du Bénin, Parakou, Bénin.

Snedecor GW. et Cochran WG: 1971. Méthodes statistiques. Association de coordination technique agricole, Paris, France.

Valdez VS: 1994. Cultuvo de Aji, Edition : Centro de Información de FDA. 